DOI: https://doi.org/10.14311/TPFM.2022.006

\title{
SIMPLE RHEOSCOPIC FLOWS USED IN TEACHING FLUID MECHANICS
}

\author{
Daniel Duda ${ }^{1}$, V. Yanovych ${ }^{1,2}$, V. Uruba ${ }^{1,2}$ \\ ${ }^{1}$ Faculty of Mechanical Engineering, University of West Bohemia in Pilsen, Univerzitní 22, \\ 30614 Pilsen, Czech Republic \\ 2 Institute of Thermomechanics, Academy of Sciences of the Czech Republic, Dolejškova 5, \\ 18000 Prague, Czech Republic
}

\begin{abstract}
Four simple demonstration experiments are presented. They are used as a support in the teaching of Fluid Mechanics I (a compulsory lecture at the University of West Bohemia in Pilsen). All mentioned experiments use the rheoscopic fluid obtained as a water solution of mica powder to visualize the flow in a esthetic way, which, as we hope, has a potential to attract students attention. The experiments are: demonstration of Bernoulli equation in a widening and narrowing channel; Taylor-Couette flow; the effect of viscosity to the scales and decay of turbulence; and the Galilean transformation inside an axial compressor.
\end{abstract}

Keywords: Teaching, Fluid mechanics, Demonstration experiment, rheoscopic fluid, Bernoulli equation, Taylor-Couette flow, Axial compressor.

\section{Introduction}

Teaching is a part of key precursors of scientific progress. Although it is indirect, its quality implies the quality of scientific research in the future. The scientific, technical and economical level of each nation in the future of few decades is determined by the quality of education now. Hence, the education has to be true and not contaminated with some ideology or politics; it has to be performed in national languages (definitely not English, which is otherwise an excellent language for sharing results and ideas among experts); it is good, when education can be for free (but this depends on the level of public finances in each country); and, last but not least, the form has to be interesting for the students to attract their attention in current age full of easily accessible entertainment. This issue we are facing at the University of West Bohemia in Pilsen.

According to our previous attempt in this direction published in conference paper [1], we observe that the students are generally clever enough to understand and to pass the exam. However, they lack of motivation - there is lot of students, who learn just the minimum to pass the exam for 3 (the worst grade allowing passing), although their abilities are higher. We think, that it is pity, because they could know more than just the basic equations. Our dream s to change the student's mind from "give me the list of equations to memorize and I will spent the minimal time with it." to "wow, show me more fascinating phenomena.".

In this conference contribution, we want to share simple experiments shown to the students to attract their attention to the fascinating nature of invisible fluid flows around us.

\subsection{Rheoscopic fluid}

The rheoscopic ${ }^{1}$ or sometimes kaliroscopic ${ }^{2}$ effect is caused by orienting of small particles according to the velocity gradient in the flow. These particles have to be strongly anisotropic (disks or scales) and shiny to reflect the light when aligned due to the velocity gradients. The interpretation of such images is not straight-forward and it is still a subject of scientific research [2].

The strain tensor rotates the anisotropic particle with moment proportional to the normal and the strain tensor, Fig. 1. Look to [2] for more details and the exact formulas. At this point, the viscosity is quite important damping the rotational inertia of the particles, which would rotate under the velocity gradient when there was no damping. The temporal development is quite complicated even when the effect of particles to the flow is neglected. Therefore, there exists no exact way how to interpret the observed pictures of rheoscopic flows.

\footnotetext{
${ }^{1}$ from Greek: $\rho \epsilon \iota \nu$ - to flow; $\sigma \kappa \mathrm{o} \pi \epsilon \iota \nu$ - to observe

${ }^{2} \kappa \alpha \lambda$ os - beautiful
} 
(a) no velocity gradient

(b) velocity gradient

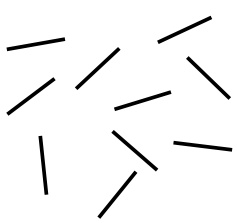

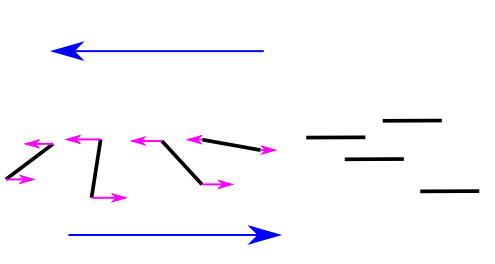

(c) coherent reflection

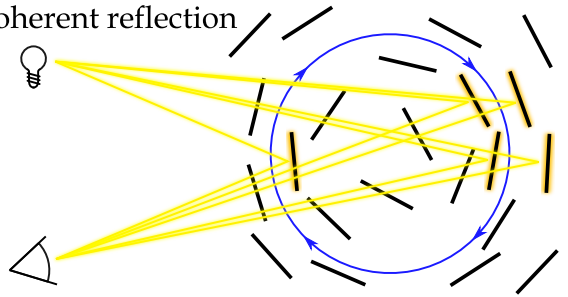

Figure 1: The rough scheme of the ordering of rheoscoopic particles: (a) the particles are randomly oriented if no moments act. (b) Under a velocity gradient, they orient normally to the gradient and (c) due to the shiny surface, they reflect light in the areas of correct coherent orientation. But this is only rough first-order view.

However, it can easily recognized, whatever the flow is turbulent or not. This property has been used in the past in many scientific observations of the flow and it helped to understand some of the mysterious properties of the turbulence transitions. E.g. Carlson et al. [3] studied plane Poiseuille flow observing the turbulent spots, which are easily recognizable. The transition between the regimes of turbulent spots has been observed by Tillmark and Alfredsson [4]. The rheoscopic flow visualization is often used in the studies of Taylor-Couette problem. Besides to the famous work of Andereck [5], the rheoscopic flow visualization has been used by Berrich et al. [6] to study the Taylor-Couette flow with imposed axial flow. On the other hand, Richter et al. [7] studied the effect of ribbed rotor in a Taylor-vortex reactor used for mixing in chemical industry. They compared ribbed and smooth cylinders and observed a immobilization of Taylor vortices caused by the ribs. Ouellette [8] used rheoscopic and electricity conducting fluid to visualize the $2 \mathrm{D}$ (1 mm thick, $1 \mathrm{~m}$ wide) homogenous and isotropic turbulence forced by Lorentz force to visualize the inverse energy cascade [9] pumping energy to large scales.

\section{Experiments}

\subsection{The Bernoulli principle}

The Bernoulli equation in incompressible fluids represents the law of energy conservation along a streamline, which leads to a well known effect, that larger velocity is connected with lower static pressure. This effect seem to be paradoxical for first look. This effect has been shown many times in simple experimental setup with water flowing through a channel with changing cross-section and the pressure is measured by the water column in tubes connected to the channel. We enriched this common setup by adding the rheoscopic fluid, which highlights the turbulence in the channel, mainly in the diverging part, where the longitudinal pressure gradient supports the boundary layer separation. This effects are responsible for a pressure loss observable within this simple system as well. Therefore, this simple gadget can be shown to the students, first, during introducing the Bernoulli equation, second, when adding the empirical pressure loss term into the Bernoulli equation.

\subsection{The Taylor-Couette flow with centrifugal Rayleigh-Taylor instability}

The Taylor-Couette flow is a widely studied case due to its simplicity (it is just a pair of concentric cylinders with gap filled with fluid in between) and due to the incredible wealth of flow regimes. These regimes were scientifically mapped by Andereck [5]. This problem is referred as a "hydrogen atom of fluid dynamics" [10].

The Taylor instability is a kind of more general Rayleigh-Taylor instability driven by the centrifugal force acting to the fluid near the inner rotating cylinder. This fluid is pushed away (in its reference frame) and replaced by the fluid from the outer part of the gap.

We already described this simple demonstration experiment in our previous conference contribution [1], although, the apparatus was not working at the deadline of that conference. Therefore, 
(a) Channel, the water flows from right to left

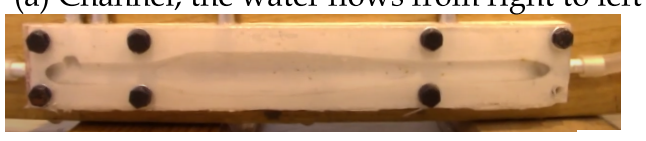

(b) Water level in the tubes
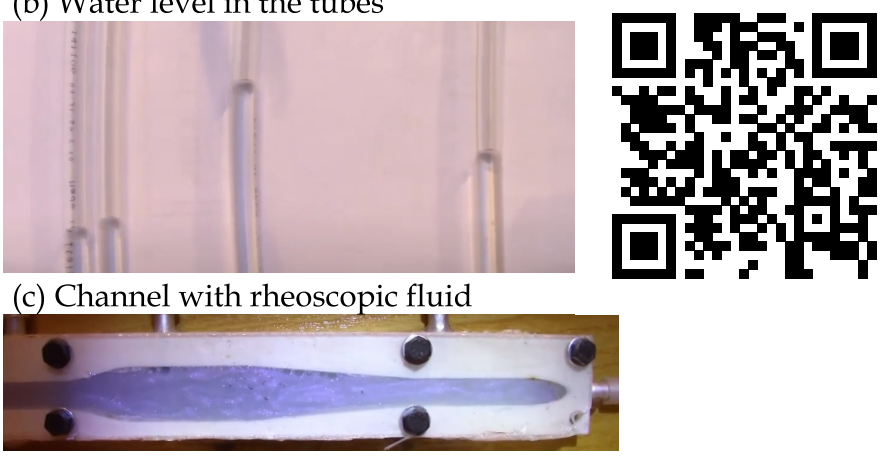

Figure 2: (a)The 3D printed channel for the flow; the flow enters at the right-hand-side and leaves the channel at the left-hand one. (b) Photograph of the tubes filled with water, the static pressure is clearly largest in the part with largest cross-section. The static pressure does not return to the level before the widening due to pressure loss. (c) The flow visualized by using rheoscopic fluid demonstrates the separation and consequent turbulence in the channel widening. The QR-code links to a movie: https://youtu.be/d0ZpAJyMzLo.

we venture to present it second times, see Fig. 3.

\subsection{The effect of viscosity to turbulence scales}

The fluid viscosity has the effect of smoothing the velocity gradients of neighboring areas of the fluid and therefore it is responsible for damping of internal motion of the fluid, the so-called dissipation [11]. Viscosity plays an important role in the Reynolds number definition [12]

$$
\operatorname{Re}=\frac{u L}{\nu}
$$

where $u$ is same characteristic velocity, $L$ some characteristic length and $\nu$ is the kinematic viscosity, which is the only parameter, which is in most cases easily and clearly obtainable from tables. The others, velocity and length, are subject of discussion, what means the word characteristic. However, in geometrically similar cases, the comparison can be made quite easily. The Reynolds number can be understood as a ratio of scales in the turbulence [12], but not linearly:

$$
\frac{L}{\eta} \approx\left(\frac{u L}{\nu}\right)^{\frac{3}{4}}
$$

in other words, larger Re signifies smaller Kolmogorov scale $\eta$ and thus more smallest vortices fit into the probed volume $[13,14]$. Oppositely, when increasing the viscosity $\nu$, the flow becomes smoother with larger (and thus better apparent) structures.

The simple presented apparatus consists of three test tubes of the same dimensions containing the same iron balls, Fig. 4. These balls are moved by permanent magnets on a common support and thus the velocity is forced to be the same in all three tubes. The only parameter, which vary, is the viscosity. A short movie shows two effects: first, the increasing viscosity leads to smoother structures in the flow. Second effect is connected with the turbulence decay: in steady-state case, the energy flows through Richardson cascade [15] from large to small scales, where it dissipates at $\eta$. But, when there is no more input, then the dissipative Kolmogorov scale $\eta$ grows with time and we observe, that the small vortices decay first leaving the large structures, which move less (they are losing energy), but alive longer. This effect is responsible for another set of paradoxes: eg. that 

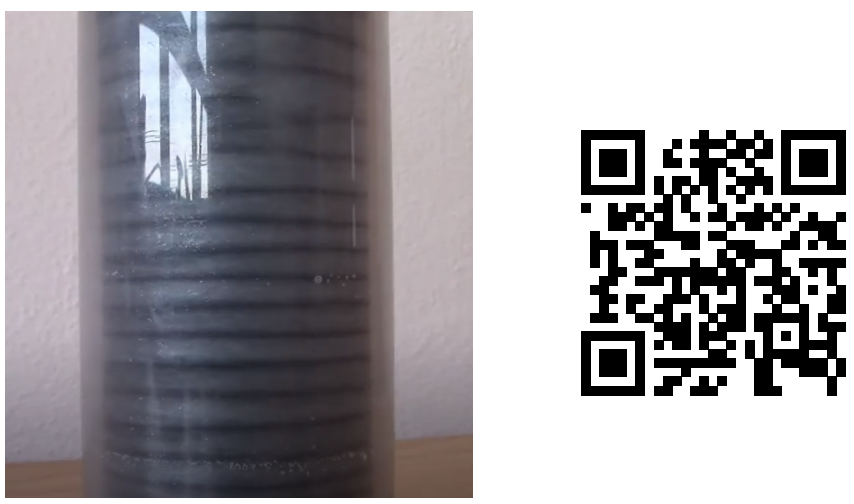

Figure 3: Taylor-Couette flow in a cylindrical cell with rotating inner cylinder. The QR-code links to a movie: https://youtu. be/hbwHOuvp2nE.

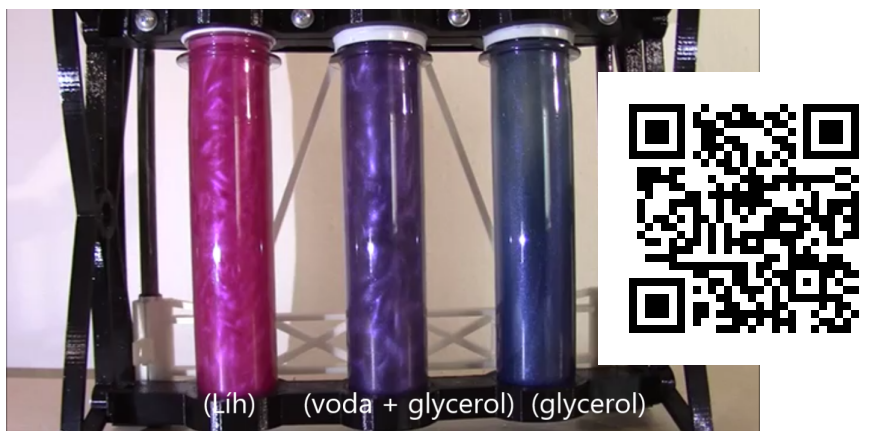

Figure 4: Three test tubes filled with different liquids, left ethanol, middle water + glycerin, right pure glycerin. All the tubes contain the same iron ball which is moved by a magnet on the white support in behind. The QR-code links to a movie: https://youtu.be/dxcRUnd9Bp8. Special thanks to Ing. Martin Pelikán for the set of iron balls of same dimensions and to Michaela Vacková for the set of PET tubes usually used as precursors of PET bottles.

adding a grid into wind tunnel settling chamber suppresses turbulence, although grids generate turbulence (the grid adds turbulence of the scale of mesh parameter and suppress turbulence on larger scales, but the mesh-scale turbulence dies faster, so the net effect is less turbulence far downstream); or as shown by Kühnen et al. [16], the turbulization at smaller scales destabilizes turbulence at larger scales and therefore the turbulence entirely decays faster.

\subsection{The Galilean transformation inside an axial flow machine}

The flow through a turbine or a compressor has a large importance in engineering practice. One of the moments difficult for students, is the Galilean transformation between the static part and the rotating part. This transformation is needed only, when we are thinking about a static snapshot, in the real moving system, the fluid just flows through the static or moving parts without some abrupt change of direction. The change of direction is only virtual and then the Galilean transformation has to be taken into account. The velocity in a moving reference frame $\vec{u}^{\prime}$ is simply the difference between the velocity in static reference frame $\vec{u}$ and the relative velocity of moving reference frame to the static reference frame $\vec{v}$

$$
\vec{u}^{\prime}=\vec{u}-\vec{v}
$$

Therefore, the velocity in direction stator blades transforms into opposite direction of rotor blades in a such way, that it enters directly into them (of course, when there are no losses).

Our next simple experiment in Fig. 5 shows the flow through an axial compressor without head (therefore, the blades are just a straight desks and the flowing fluid does not produce forces). 


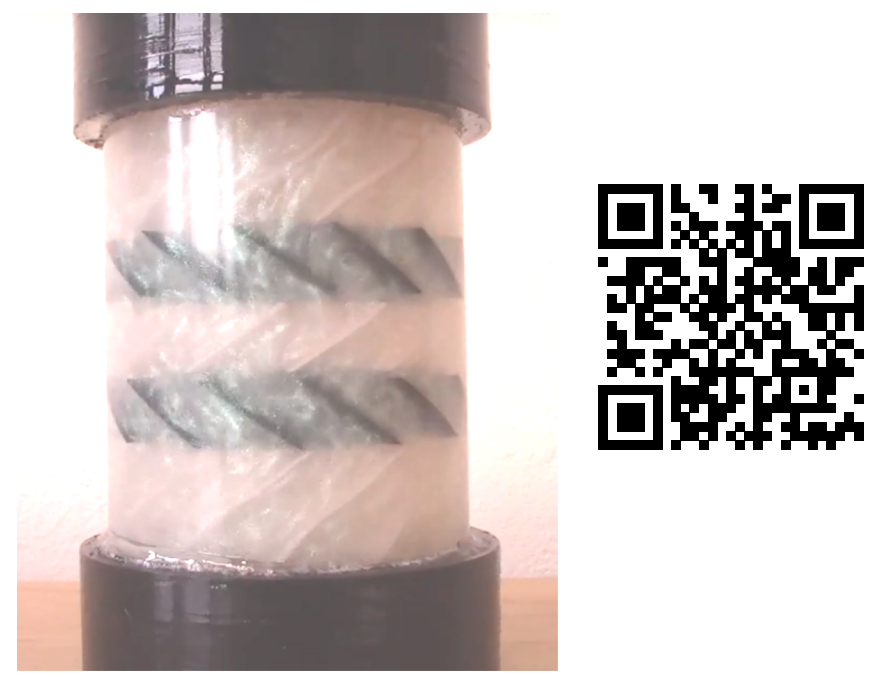

Figure 5: Axial compressor in a transparent plexiglass tube moved by hand. The QR-code links to a movie: https://youtu.be/DHj101R265M. Special thanks to Michaela Vacková for the plexiglass tube.

The best feeling is obtained, when You could touch it personally and see, how the axial velocity instantly reacts to the angular velocity of shaft moved simply by hand. However, the velocity fields drawn in the textbooks is just a first assumption, the higher-order effects include turbulence flows around the blades, which is visualized by added rheoscopic fluid. A more exact measurement of the flow inside an axial turbine has been published in [17].

\section{Discussion}

In this section we want to share few practical tips for easier repetition of our simple experiments.

There are various techniques to get rheoscopic fluid. The historical experiments were performed by using aluminum or graphite powder. Borrero-Echeverry and co-workers suggest stearic acid crystals obtained from shaving cream [18] (we did not try yet). Our experiments limit to mica, which is easily accessible, although it has some limiting properties. Mica is used in cosmetics, thus the easiest way to obtain it is to buy it in a perfumery. When mica stays in water for several hours, the powder will coagulate losing its nice rheoscopic properties. To avoid this, the best solution is to add ethanol. The hydrogen peroxide can be used to avoid growth of algae and cyanobacteria in the case, that ethanol is not applicable. Ethanol helps during mica mixing as well - it slightly decreases the surface tension, thus the powder wets more easily. It is also practical, to mix the mica in a small amount of liquid, and then add this "concentrate" into larger volume.

It is not possible to use ethanol, when the vessel is made from plexiglass (Poly(methyl methacrylate)), because ethanol is dilutable in this polymer, which increases the inner tension, makes it brittle and completely destroys the vessel in few minutes. We have found a transparent polystyrene desks, which are very well applicable as a covering of some experiment. Unfortunately, there are no tubes made of transparent polystyrene, they are made from plexiglass only.

The concentration of particles is another "alchemical" issue. The concentration controls the depth, where the flow is observable. Roughly speaking, the concentration and thus the transparent depth, should be adapted to the length-scale of turbulence, which would be observed. At each wall, even the transparent smooth wall, there is a boundary layer with its own internal strain and turbulent structures. If there will be too many particles, the bulk flow will be hidden behind the boundary layer. Fortunately, the typical boundary layer thickness is very thin in the discussed cases.

3D print is a great tool for producing such a small-amount gadgets. However, we have found, that the $3 \mathrm{D}$ printed vessels are not entirely watertide - the water can leak between layers. Painting 
the model with a transparent Nitrocellulose lack solves this issue.

\section{Acknowledgment}

The work was supported from ERDF under project Research Cooperation for Higher Efficiency and Reliability of Blade Machines (LoStr) No. CZ.02.1.01 / 0.0 / 0.0 / 16_026 / 0008389.

We thank to Michaela Vacková for paying the conference fee.

\section{References}

[1] Duda, D. \& Klimko, M. \& Škach, R. \& Uher, J., \& Uruba, V.: Hydrodynamic education with rheoscopic fluid. EPJ Web Conf., vol. 213 (2019) p. 5.

[2] WILKINSON, M. \& BEZUGLYY, V., \& MEHLIG, B.: Emergent order in rheoscopic swirls. Journal of Fluid Mechanics, vol. 667 (2011) pp. 158-187.

[3] Carlson, D. R. \& Widnall, S. E., \& Peeters, M. F.: A flow-visualization study of transition in plane poiseuille flow. Journal of Fluid Mechanics, vol. 121 (1982) pp. 487-505.

[4] Tillmark, N. \& Alfredsson, P. H.: Experiments on transition in plane couette flow. Journal of Fluid Mechanics, vol. 235 (1992) pp. 89-102.

[5] Andereck, C. D. \& Liu, S. S., \& Swinney, H. L.: Flow regimes in a circular couette system with independently rotating cylinders. Journal of Fluid Mechanics, vol. 164 (1986) pp. 155-183.

[6] On the Stability of Taylor - Couette Flow With Axial Flow vol. Volume 1: Symposia, Parts A and B of Fluids Engineering Division Summer Meeting (2012).

[7] Richter, O. \& Hoffmann, H., \& Kraushaar-Czarnetzki, B.: Effect of the rotor shape on the mixing characteristics of a continuous flow taylor-vortex reactor. Chemical Engineering Science, vol. 63 (2008) pp. 3504-3513.

[8] Ouellette, N. T.: Turbulence in two dimensions. Physics Today, vol. 65 no. 5: (2012) pp. 68-69.

[9] Kraichnan, R. H.: Inertial ranges in two?dimensional turbulence. Physics of Fluids, vol. 10 (1967).

[10] Fardin, M. \& Perge, C., \& Taberlet, N.: The hydrogen atom of fluid dynamics - introduction to the taylor-couette flow for soft matter scientists. Soft Matter, vol. 10 (2014) pp. 3523-3535.

[11] Alexakis, A. \& Biferale, L.: Cascades and transitions in turbulent flows. Physics Reports, vol. 767-769 (2018) pp. 1-101.

[12] Uruba, V.: On reynolds number physical interpretation. AIP Conference Proceedings, vol. 2000 no. 1: (2018) p. 020019.

[13] Duda, D. \& Uruba, V.: Spatial spectrum from particle image velocimetry data. ASME J of Nuclear Rad Sci., vol. 5 no. 030912: (2019).

[14] Duda, D. \& Uruba, V.: Piv of air flow over a step and discussion of fluctuation decompositions. AIP Conference Proceedings, vol. 2000 (2018).

[15] Richardson, L. F.: Atmospheric diffusion shown on a distance-neighbour graph. Proceedings of the Royal Society A: Mathematical, Physical and Engineering Sciences, vol. 10 no. 756: (1926) pp. 709-737.

[16] Kühnen, J. \& Song, B. \& Scarselli, D. \& Budanur, N. B. \& Riedl, M. \& Willis, A. P. \& Avila, M., \& Hof, B.: Destabilizing turbulence in pipe flow. Nature Physics, vol. 14 (2018) pp. 386-390. 
[17] Duda, D. \& Jelínek, T. \& Milčák, P. \& Němec, M. \& Uruba, V. \& Yanovych, V., \& Žitek, P.: Experimental investigation of the unsteady stator/rotor wake characteristics downstream of an axial air turbine. International Journal of Turbomachinery, Propulsion and Power, vol. 6 no. 3: (2021).

[18] Borrero-Echeverry, D. \& Crowley, C. J., \& Riddick, T. P.: Rheoscopic fluids in a postkalliroscope world. Physics of Fluids, vol. 30 (2018). 